\title{
Serious infections in patients with self- reported psoriatic arthritis from the Psoriasis Longitudinal Assessment and Registry (PSOLAR) treated with biologics
}

Christopher T. Ritchlin ${ }^{1 *}$, Mona Stahle ${ }^{2}$, Yves Poulin ${ }^{3}$, Jerry Bagel ${ }^{4}$, Soumya D. Chakravarty ${ }^{5,6}$, Shelly Kafka ${ }^{5}$, Bhaskar Srivastava ${ }^{5}$, Wayne Langholff ${ }^{7}$ and Alice B. Gottlieb ${ }^{8}$

\begin{abstract}
Background: Patients with psoriatic arthritis (PsA) have increased risk of adverse events, including serious infections (SI), compared with psoriasis patients.

Methods: Patients eligible for, or receiving conventional systemic and biologic agents for psoriasis were followed prospectively using PSOLAR. Cohorts included: ustekinumab, tumor necrosis factor (TNF) inhibitors; infliximab; etanercept; adalimumab; non-biologic/methotrexate (MTX) (reference group); and non-biologic/non-MTX. Multivariate analyses using Cox hazard regression were used to identify factors associated with time to first SI. Rates of SI in PSOLAR psoriasis patients with self-reported PsA and possible risks with biologic therapy were evaluated.

Results: PSOLAR enrolled 4315 psoriasis patients with self-reported PSA. The overall population $(N=2401)$ included patients (n): 628 ustekinumab; 1413 TNF inhibitors; 258 infliximab; 481 etanercept; 674 adalimumab; 54 other biologics, 98 non-biologic/MTX; 208 non-biologic/non-MTX. Overall, 138 SI were reported with incidence rates per 100 patient-years as follows: a) ustekinumab: 1.00; b) TNF inhibitors: 2.22; c) infliximab: 2.12; d) etanercept: 2.58; e) adalimumab: 1.99; f) non-biologic/MTX: 3.01; g) and non-biologic/non-MTX: 2.31. Age, time-dependent disease activity Physician's Global Assessment (PGA) of 4, 5, history of infection, and diabetes were associated with increased risk for SI $(p<0.05)$ in self-reported PsA patients. Biologic groups, other than ustekinumab, had numerically higher rates of SI.

Conclusions: PSOLAR psoriasis patients with self-reported PSA in the TNF inhibitors, infliximab, adalimumab, etanercept, and MTX cohorts had numerically higher SI rates than the ustekinumab cohort, although not statistically significant. Age, PGA 4, 5, history of infection, and diabetes were associated with an increased risk for Sl, irrespective of biologic exposure.
\end{abstract}

Trial registration: NCT00508547; Registered July 30, 2007.

Keywords: PSOLAR, Psoriasis, Psoriatic arthritis, Serious infections

\footnotetext{
* Correspondence: Christopher_Ritchlin@URMC.Rochester.edu

${ }^{1}$ Division of Allergy, Immunology, and Rheumatology, University of Rochester

Medical Center, 601 Elmwood Ave., Box 695, Rochester, NY 14642, USA

Full list of author information is available at the end of the article
}

(c) The Author(s). 2019 Open Access This article is distributed under the terms of the Creative Commons Attribution 4.0 International License (http://creativecommons.org/licenses/by/4.0/), which permits unrestricted use, distribution, and reproduction in any medium, provided you give appropriate credit to the original author(s) and the source, provide a link to the Creative Commons license, and indicate if changes were made. The Creative Commons Public Domain Dedication waiver (http://creativecommons.org/publicdomain/zero/1.0/) applies to the data made available in this article, unless otherwise stated. 


\section{Background}

Psoriatic arthritis (PsA) is an immune-mediated inflammatory disease that typically involves inflammation of the peripheral joints, enthesitis, dactylitis, spondylitis, and psoriasis [1]. PsA can be aggressive and therefore result in decreased function in daily activities, impaired health-related quality of life, and increased mortality [1-3]. Due to the chronic inflammatory nature of this disease, patients often require long-term treatment. Several studies have reported increased risk of infections with biologic use in patients with psoriasis [2, 4-7] and rheumatoid arthritis (RA) [2, 8-12]. Likewise, patients with PsA have been reported to have an increased risk of comorbidities and adverse events (AEs), including serious infections (SIs), compared with patients with psoriasis [13, 14]. Many investigators question whether the increased risk for AEs is due to the underlying inflammatory nature of psoriatic disease itself, or to the therapies used to treat the disease.

The impact of biologic use and infection risk is an area of significant interest to the rheumatology community. An unmet need remains for longitudinal data to better address risks for SIs in patients identified as having PsA in routine clinical practice. Notably, the majority of available data regarding the impact of biologic therapies on SI risk has been directed at other inflammatory arthritides, such as RA $[15,16]$, and less so for patients with PsA [17]. Consequently, an unmet need remains to assess the risk for SI in the PsA population and potentially identify any differences between outcomes of exposure in real-world use that may exist. The Psoriasis Longitudinal Assessment and Registry (PSOLAR), an observational psoriasis registry with more than 12,000 patients, captures safety data among patients treated with biologics and/or systemic agents and can help address this real-world need.

The risk of SIs in patients with psoriasis from the PSOLAR registry has been previously reported [7]. In this report, we evaluated the incidence of SIs in a subpopulation of patients with self-reported PsA enrolled in the psoriasis PSOLAR registry [18], and specifically, among patients receiving biologic therapies for their psoriasis with concomitant PsA compared with patients receiving nonbiologic therapies. PSOLAR is an international, disease-based, observational study in which patients eligible for, or receiving, conventional systemic and biologic agents for the treatment of psoriasis are followed prospectively [18]. Predictors of time to first SI were also evaluated. This is the first modeled analysis of patients with selfreported PsA enrolled in PSOLAR, the largest psoriasis registry to date.

\section{Methods}

\section{Patients and study design}

The PSOLAR patient populations and study design have been previously reported [18]. Briefly, PSOLAR enrolled adult patients ( $>18$ years) receiving, or eligible to receive, treatment with biologics and/or conventional systemic agents for psoriasis [18, 19]. As of August 23, 2015, PSOLAR was fully enrolled with 12,090 patients.

In the current report, all patients had psoriasis, including a subset of which self-reported a diagnosis of PsA $(N=4315)$ [20]. Of the patients self-reporting PsA, a subset self-reported that their healthcare provider established a diagnosis of PsA $(N=1719)$, but with no further confirmation by the investigator [20]. Patients were enrolled at international dermatology sites including academic centers, hospitals, and community practices [18]. Demographic and psoriasis disease characteristics, medical, social, and family histories, and previous medication use were collected at each site using electronic case report forms (eCRF). Data were collected at site visits at baseline and every-6-months, except medical, social, and family histories, which were only collected at baseline.

PSOLAR is conducted in accordance with the International Conference on Harmonizing guidelines on Good Clinical Practices and the Declaration of Helsinki. An institutional review board or ethics committee (Goodwyn Institutional Review Board and Ontario Institutional Review Board) approved the registry protocol. All patients provided written informed consent. This study adheres to the STROBE guidelines for observational studies.

In this report, patients were stratified by exposure to specific biologics or non-biologics. Specifically, patients were evaluated using the following treatment cohorts: ustekinumab, infliximab, adalimumab, etanercept, all other biologics, non-biologic/methotrexate (MTX) and non-biologic/non-MTX. The specific tumor necrosis factor (TNF) inhibitors evaluated in this analysis (infliximab, adalimumab, and etanercept) were selected because they were the most prevalently used in the PSOLAR registry. The "all other biologics" cohort included patients receiving a biologic indicated for PsA (e.g., golimumab, certolizumab, secukinumab), that was being studied in PsA but not yet approved at the time (e.g., abatacept, brodalumab, ixekizumab), or that is no longer commercially available (e.g., efalizumab, alefacept). The "all other biologics" cohort has been removed from all tables presented in this report due to low numbers, but is still included in the total. Patients had received one of these agents when registered in PSOLAR or began treatment after PSOLAR registration. These patients may also have been exposed in the past to a different biologic, topical psoriasis therapy, or phototherapy. Previous 
use or current exposure was allowed for MTX, but not for other systemic immunomodulators. Non-biologic/ non-MTX could have included, but were not limited to, cyclosporine, tacrolimus, mycophenolate mofetil, azathioprine, oral corticosteroids, and psoralen plus ultraviolet A, or ultraviolet B phototherapy. Patients in cohorts other than the non-biologic/non-MTX group were excluded if oral corticosteroids were used. This was done to allow for more fair comparisons and to prevent confounding the SI based on the risk of the biologic itself. For each comparison, the MTX-exposed patient cohort served as the reference group.

The cohorts described above were analyzed in three populations as follows. The overall population included prevalent (previous) users of biologics (receiving a biologic at the time of registry enrollment) and incident (new) users of biologics (starting a biologic at the time of, or after, enrollment). The incident biologic user subpopulation included only new users of a biologic at or after enrollment as defined above. Prior usage of any biologic other than the newly initiated biologic was allowed among incident users. The bionaive subpopulation included users who had never been exposed to any biologic. The primary analysis for this study was based on the overall population.

Index date (initiation of follow-up to record person years) for the cohorts was the date of registration into PSOLAR for those on current therapy OR the start date of therapy after registration for new users. Incident rates of SI were calculated based upon person years of exposure. Follow-up began on the index date until: 1) death; 2) withdrawal from the registry; 3) last data cut (August 2016); 4) 91 days after discontinuation of treatment; or 5) switching of treatment. Patients could not qualify for other treatment cohorts if switching of therapy occurred.

The exposure period for the new biologic users (incident user and bionaive subpopulations) started at the date of the first cohort-defining biologic dose on registry and ended at the earlier of 90 days after the last cohortdefining biologic dose, the date of switching to a different biologic, the date of starting other systemic immunomodulators, discontinuation from the registry, registry data cut (August 23, 2016), or death. The exposure period for prevalent biologic users started at registry enrollment and ended as described for the new biologic users. Only the first cohort-defining biologic was considered; biologic usage after switching was excluded.

Both non-biologic/MTX and non-biologic/non-MTX cohorts were defined the same for both prevalent and incident users. Exposure for the non-biologic/MTX was calculated from the later date of the enrollment date and the first MTX dose date and ended as the earlier of 90 days after the last MTX dose, the date of switching to other systemic immunomodulators, discontinuation from the registry, registry data cut (August 23, 2016), or death.

Exposure for the non-biologic/non-MTX starts from the later time of enrollment date and the start date of other immunomodulators on registry and ended at the earlier of 90 days after the last cohort defining drug dose, the date of switching to MTX, discontinuation from the registry, registry data cut (August 23, 2016), or death.

\section{Outcome measures}

The outcome included the number of SI events, defined as serious adverse events (SAEs) classified as "Infections and Infestations" based on the Medical Dictionary for Regulatory Activities (MedDRA) coding system. SAEs are defined as any undesirable experience associated with the use of a medical product in a patient that may result in death, be life-threatening, lead to hospitalization or prolonged hospitalization, disability or permanent damage, congenital anomaly or birth defect, or require intervention to prevent permanent impairment or damage. All AEs were identified by the site investigator, collected through eCRF, and reviewed. Verbatim terms provided by sites are coded to MedDRA terms and are subject to verification by site monitors. All SAEs regardless of exposure are subject to verification by study personnel. Incomplete reports are queried for context to verify the event. This is performed by the study nurse and physician initially and cross-checked for completeness by the sponsor's medical monitor. Queries are generated if the rationale/context of the reported event term is unclear. Despite monitoring of serious event documentation, source documents may not necessarily be obtainable for all diagnoses. Nonetheless, reporting, categorization and definition of serious infections is designed to be non-differential across all treatment cohorts since the study support team performs uniform follow-up on all cases. History of infections was defined as infections requiring a prescription medication within 3 years of enrollment. Rates of multiple infections and modeled analyses were based on time to first infection.

\section{Statistical analysis}

All patients from the PSOLAR registry at the time of this analysis (August 23, 2016) who self-reported having PsA were categorized into a treatment cohort based on the definitions mentioned above while excluding patients violating the rules. Baseline demographics and disease characteristics were summarized for each cohort group without imputation for missing values. Cumulative incidence rates for SI were calculated based on 100 patientyears. Cox regression models were used to identify predictors to first SI event and to estimate adjusted hazard ratios and 95\% confidence intervals (CI) of SI event. Pre- 
determined covariates at baseline (defined as the last non-missing value that is closest and prior to/on the cohort start date) were included in the multivariate model (Fig. 1). In case of missing continuous covariates, they were imputed with population mean and the imputed values were used in the model.

\section{Results}

\section{Baseline demographics and disease characteristics}

A total of 12,090 patients with psoriasis were enrolled in the PSOLAR registry at the time of this analysis. Overall, approximately $36 \%(n=4315)$ of the psoriasis patients in PSOLAR self-reported having PsA $[7,20]$.

The overall PsA population included a total of 2401 patients (7244 patient years [PY]), of which 628 had received ustekinumab; 1413 received TNF inhibitors; 258 received infliximab; 481 received etanercept, 674 received adalimumab, 98 received non-biologic/MTX, and 208 received non-biologic/non-MTX (Table 1). Biologic exposure for each group started at exposure to the first biologic therapy received on registry. The incident population included 1163 patients (3101 PY), and the bionaive population included 532 patients (1626 PY) (Table 2). In the overall population, the demographic and disease characteristics were generally comparable across therapeutic cohorts (Table 1). In general, the ratio of men to women was comparable, with a mean age of approximately 50 years. The majority of patients were white $(83.3 \%)$ and overweight $(84.6 \%)$, which typically are common findings in patients with psoriasis and PsA. All patients evaluated in this study self-reported a diagnosis of PsA, and high proportions of patients had a medical history of other comorbidities including: cardiovascular, psychiatric, endocrine, and pulmonary disorders (Table 1). Additionally, more than one-quarter of all patients in the overall PsA population (26.7\%) had a history of infections, requiring prescription medication within the last 3 years, with the infliximab (32.6\%) cohort having a slightly higher rate. Baseline characteristics for the incident (Additional file 1: Table S1) and bionaive

Subgroup
Age/10 years at baseline ${ }^{b}$
Men
Non-White
Overweight/Obesity class I at baseline
Obesity class II, III at baseline
Duration of psoriasis/5 years at baseline
Diabetes mellitus type I, II
History of infections ${ }^{c}$
History of $2+$ biologics
PGA 2, 3 at baseline
PGA 4, 5 at baseline
Time-dependent disease activity PGA 2, 3
Time-dependent disease activity PGA 4,5
Smoking (current vs. past/never)
Ustekinumab
TNF inhibitors
Infliximab
Etanercept
Adalimumab
Non-biologic/non-MTX

0.1
Hazard ratio $(95 \% \mathrm{Cl})$

$2,3^{d}$

\section{4,5}

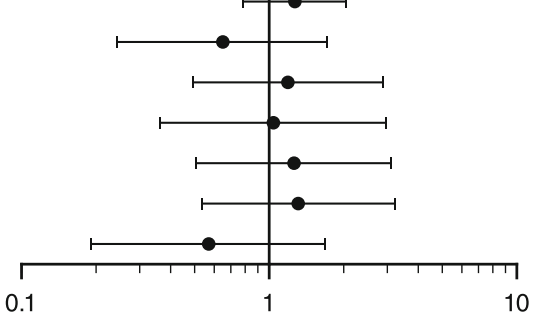

a Hazard ratios are derived using Cox proportional model with other covariates adjusted.

b Baseline is defined as the last non-missing value that is closest and prior to/on the start date of the cohort defining biologic/therapy.

c Requiring prescription medication within the last 3 years.

d Time-dependent disease activity $(P G A)$ is the non-missing value from the last visit prior to the first serious infection event or prior to the cohort defining therapy end date.

e TNF inhibitors includes infliximab, etanercept, and adalimumab.

Historical peak PGA 2, 3 and Historical peak PGA 4, 5 were included in the multivariate analyses, but are not displayed.

Fig. 1 Predictors of time-to-first serious infection in PSOLAR psoriasis patients with self-reported PsA (overall population). Cl, confidence interval; MTX, methotrexate; PGA, Physician's Global Assessment; PsA, psoriatic arthritis; PSOLAR, Psoriasis Longitudinal Assessment and Registry; TNF, tumor necrosis factor 


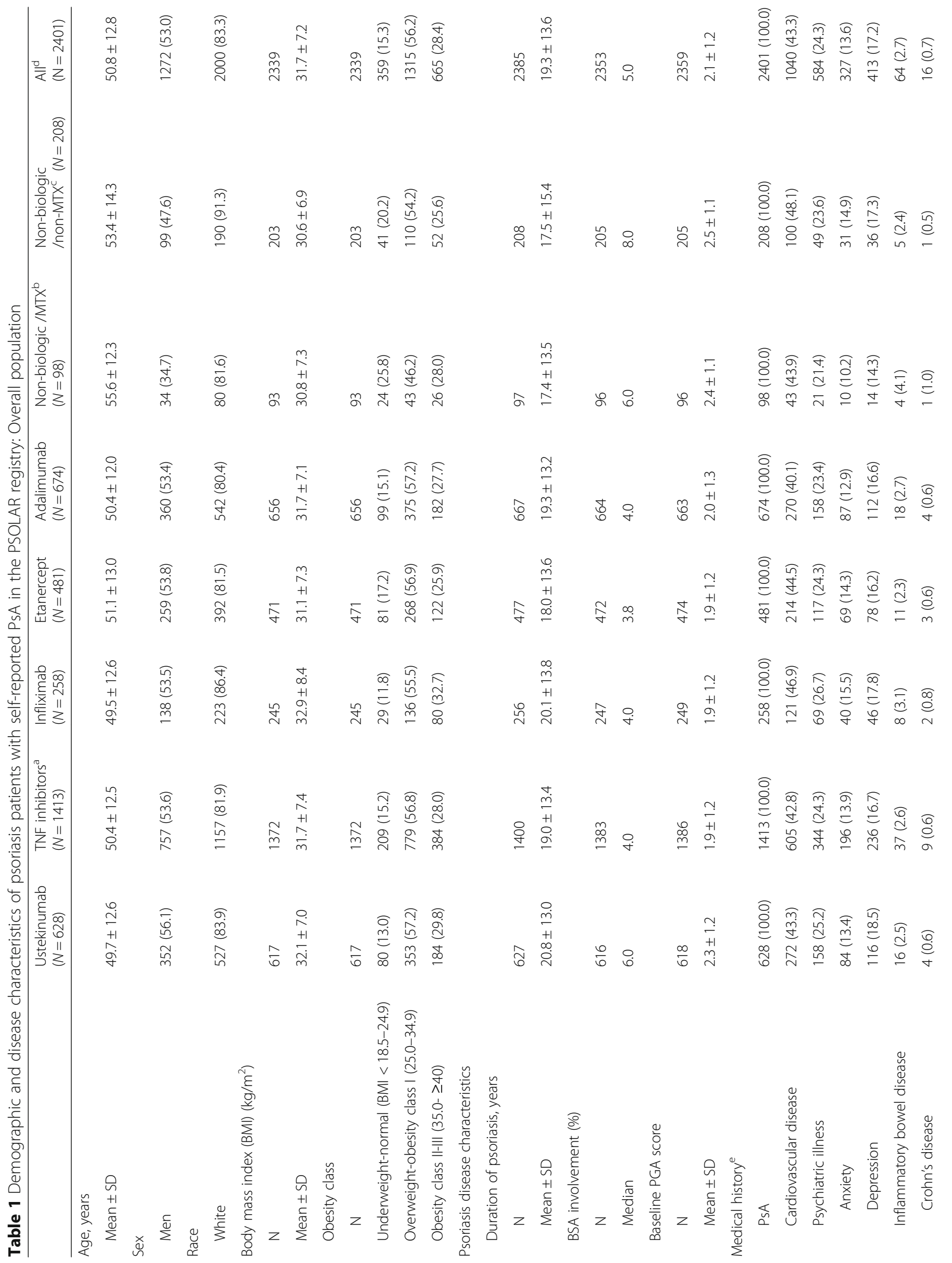




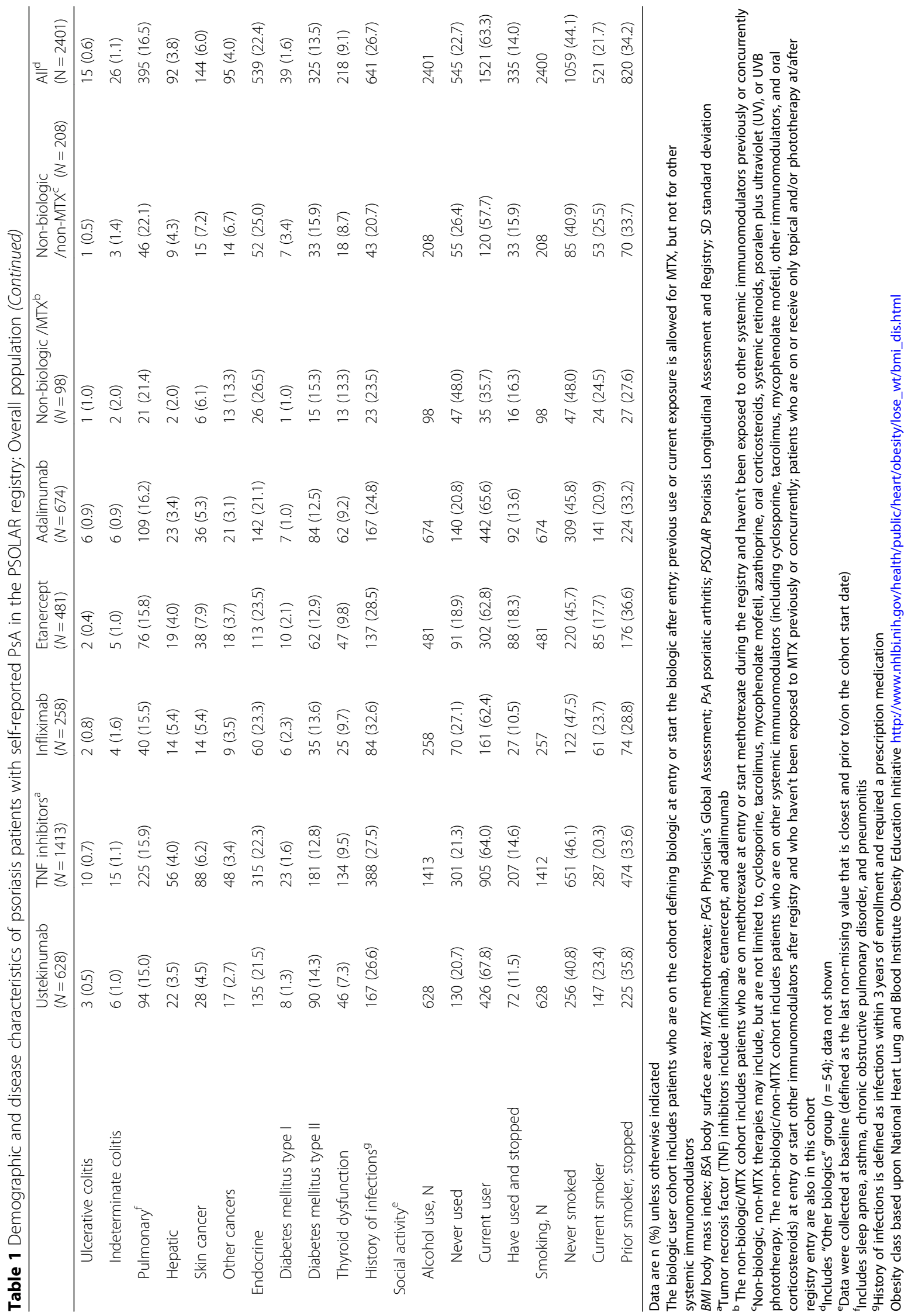


Table 2 Number of patient-years of follow-up by population and treatment cohort in psoriasis patients with self-reported PsA enrolled in PSOLAR

\begin{tabular}{|c|c|c|c|c|c|c|c|c|}
\hline & Ustekinumab & TNF inhibitors $^{a}$ & Infliximab & Etanercept & Adalimumab & $\begin{array}{l}\text { Non-biologic/ } \\
\text { MTX }^{b}\end{array}$ & $\begin{array}{l}\text { Non-biologic/ } \\
\text { non-MTX }\end{array}$ & $A l^{d}$ \\
\hline \multicolumn{9}{|c|}{ Overall population } \\
\hline N & 628 & 1413 & 258 & 481 & 674 & 98 & 208 & 2401 \\
\hline Patient-years & 2009 & 4101 & 756 & 1432 & 1913 & 366 & 694 & 7244 \\
\hline \multicolumn{9}{|c|}{ Duration of follow-up, years } \\
\hline Mean \pm SD & $5.0 \pm 1.9$ & $4.9 \pm 2.2$ & $4.7 \pm 2.3$ & $4.8 \pm 2.3$ & $5.0 \pm 2.1$ & $4.2 \pm 2.2$ & $3.7 \pm 2.6$ & $4.8 \pm 2.2$ \\
\hline Median & 5.16 & 5.16 & 4.70 & 5.16 & 5.35 & 4.32 & 3.50 & 5.11 \\
\hline \multicolumn{9}{|c|}{ Incident population } \\
\hline N & 326 & 486 & 64 & 130 & 292 & 98 & 208 & 1163 \\
\hline Patient-years & 904 & 1082 & 125 & 291 & 665 & 366 & 694 & 3101 \\
\hline \multicolumn{9}{|c|}{ Duration of follow-up, years } \\
\hline Mean \pm SD & $5.4 \pm 1.9$ & $5.4 \pm 2.0$ & $5.3 \pm 2.0$ & $5.5 \pm 2.0$ & $5.4 \pm 2.0$ & $4.2 \pm 2.2$ & $3.7 \pm 2.6$ & $5.0 \pm 2.2$ \\
\hline Median & 5.71 & 5.61 & 5.67 & 5.64 & 5.59 & 4.32 & 3.50 & 5.40 \\
\hline \multicolumn{9}{|c|}{ Bionaive population } \\
\hline N & 56 & 167 & 14 & 72 & 81 & 98 & 208 & 532 \\
\hline Patient-years & 144 & 419 & 35 & 180 & 204 & 366 & 694 & 1626 \\
\hline \multicolumn{9}{|c|}{ Duration of follow-up, years } \\
\hline Mean \pm SD & $4.4 \pm 2.0$ & $5.2 \pm 2.0$ & $4.6 \pm 1.8$ & $5.6 \pm 2.2$ & $4.8 \pm 1.9$ & $4.2 \pm 2.2$ & $3.7 \pm 2.6$ & $4.4 \pm 2.3$ \\
\hline Median & 4.66 & 5.52 & 4.39 & 6.17 & 5.14 & 4.32 & 3.50 & 4.70 \\
\hline
\end{tabular}

The biologic user cohort includes patients who are on the cohort defining biologic at entry or start the biologic after entry; previous use or current exposure is allowed for MTX, but not for other systemic immunomodulators

MTX methotrexate; PsA, psoriatic arthritis; PSOLAR, Psoriasis Longitudinal Assessment and Registry; SD, standard deviation

${ }^{a}$ Tumor necrosis factor (TNF) inhibitors include infliximab, etanercept, and adalimumab

${ }^{\mathrm{b}}$ The non-biologic/MTX cohort includes patients who are receiving MTX at entry or start methotrexate during the registry and haven't been exposed to other systemic immunomodulators previously or concurrently

'Non-biologic, non-MTX therapies may include, but are not limited to, cyclosporine, tacrolimus, mycophenolate mofetil, azathioprine, oral corticosteroids, systemic retinoids, psoralen plus ultraviolet (UV), or UVB phototherapy. The non-biologic/non-MTX cohort includes patients who are receiving other systemic immunomodulators (including cyclosporine, tacrolimus, mycophenolate mofetil, other immunomodulators, and oral corticosteroids) at entry or start other immunomodulators after registry and who haven't been exposed to MTX previously or concurrently; patients who receive only topical and/or phototherapy at/ after registry entry are also in this cohort

"Includes "Other biologics" group $(n=54)$; data not shown

(Additional file 2: Table S2) populations were generally comparable to those in the overall population.

\section{Prior medication use}

In the overall population, patients in the non-biologic/ non-MTX cohort had the greatest use of systemic steroids (23.1\%) (Table 3). MTX was the most commonly used immunomodulator among all biologic user cohorts, with the greatest reported used (60.1\%) in the infliximab cohort. As expected among psoriasis patients, topical therapies were used in the majority of patients. Overall, $77.8 \%$ of all patients had a history of biologics use, with $41.5 \%$ having used only one biologic (Table 3 ).

\section{Incidence rates of SI}

In the overall population, among patients receiving specific biologics, the rates of SIs were highest in the etanercept (2.58), infliximab (2.12), and adalimumab (1.99) cohorts, respectively (Table 4). The ustekinumab cohort had a numerically lower incidence rate (1.00) among the biologics tested individually and when compared with the rates reported in the two non-biologics cohorts. No SI events were reported in the "all other biologics" cohort (data not shown). The incident rates of SIs in the incident user were similar to those reported in the overall population; however, the number of patients in the bionaive population was too low to compare with the overall population (Table 4).

In the overall population, the incidence rate of SI was 1.91 (95\% CI: $1.60,2.25 ; n=138)$ per 7244 PY of exposure (Table 5). The SIs listed by type of infection that occurred at least two or more times across treatment cohorts were captured in Table 5. The highest incidences rate of SI among biologic users were observed in the etanercept $(2.58 ; n=37)$, TNF inhibitors (2.22; $n=91)$, and infliximab $(2.12 ; n=16)$ cohorts, while the ustekinumab cohort had the lowest incidence rate of SI $(1.00 ; n=20)$. Cellulitis $(n=25)$ and pneumonia $(n=24)$ were the most commonly 


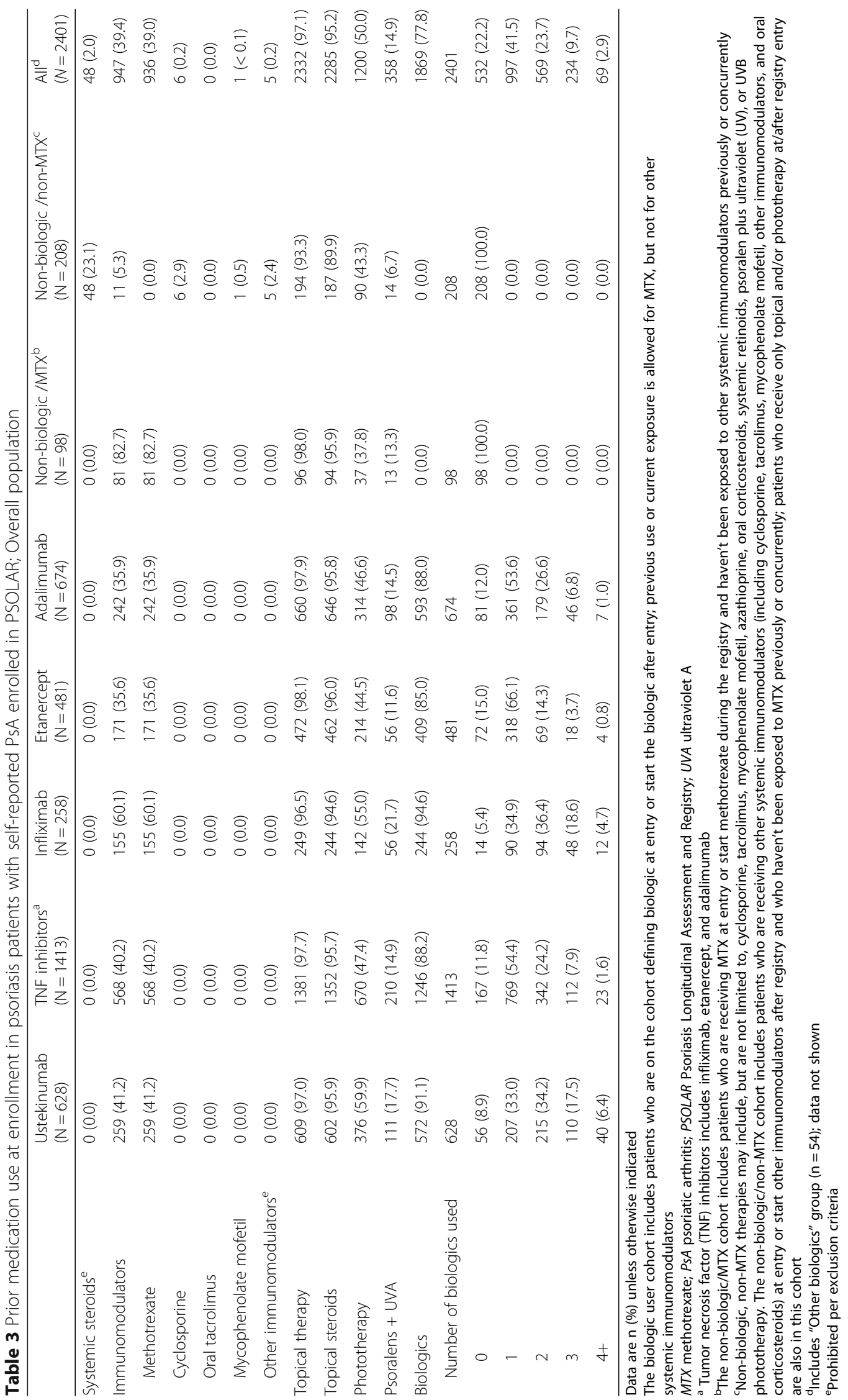




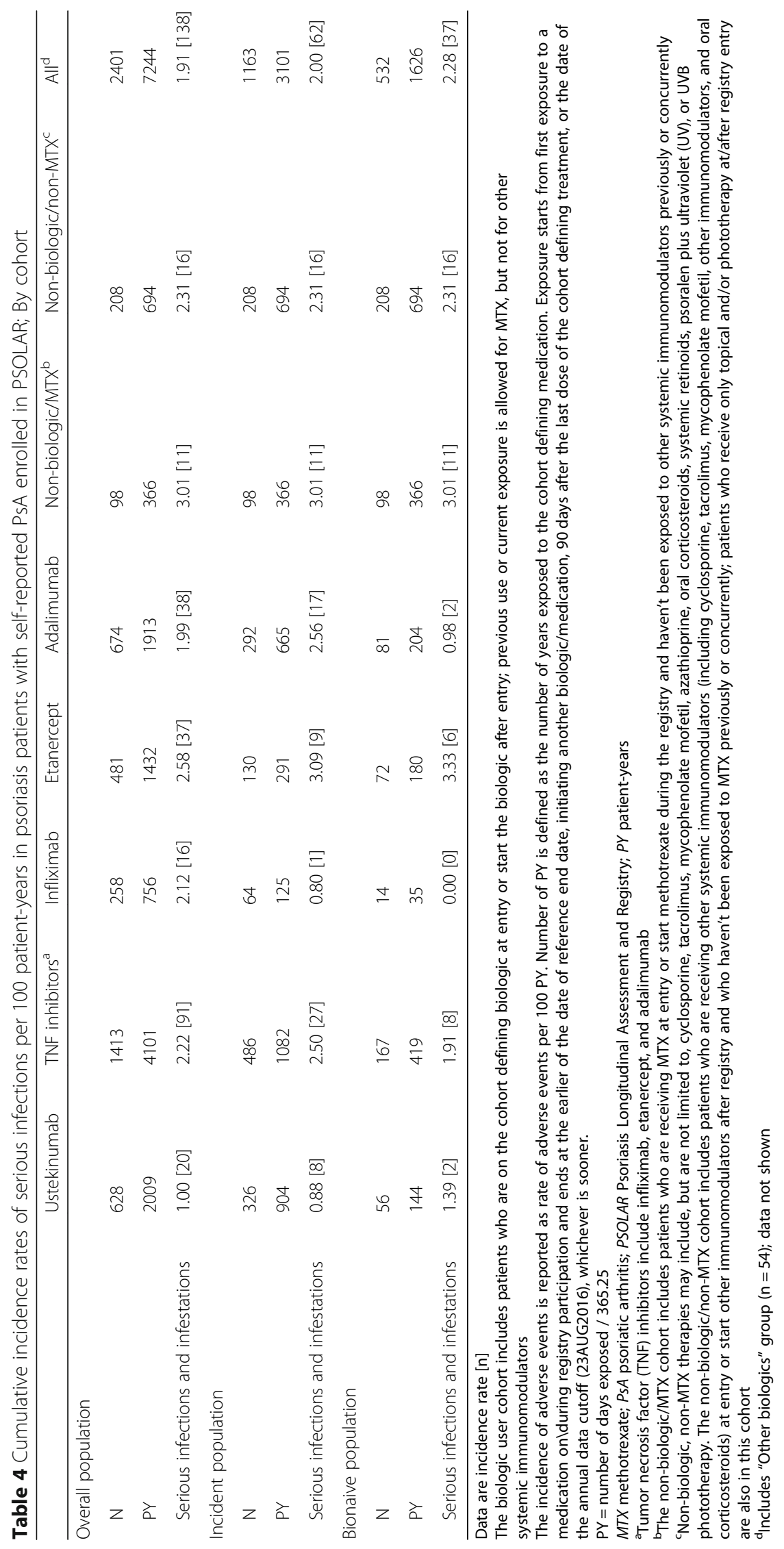




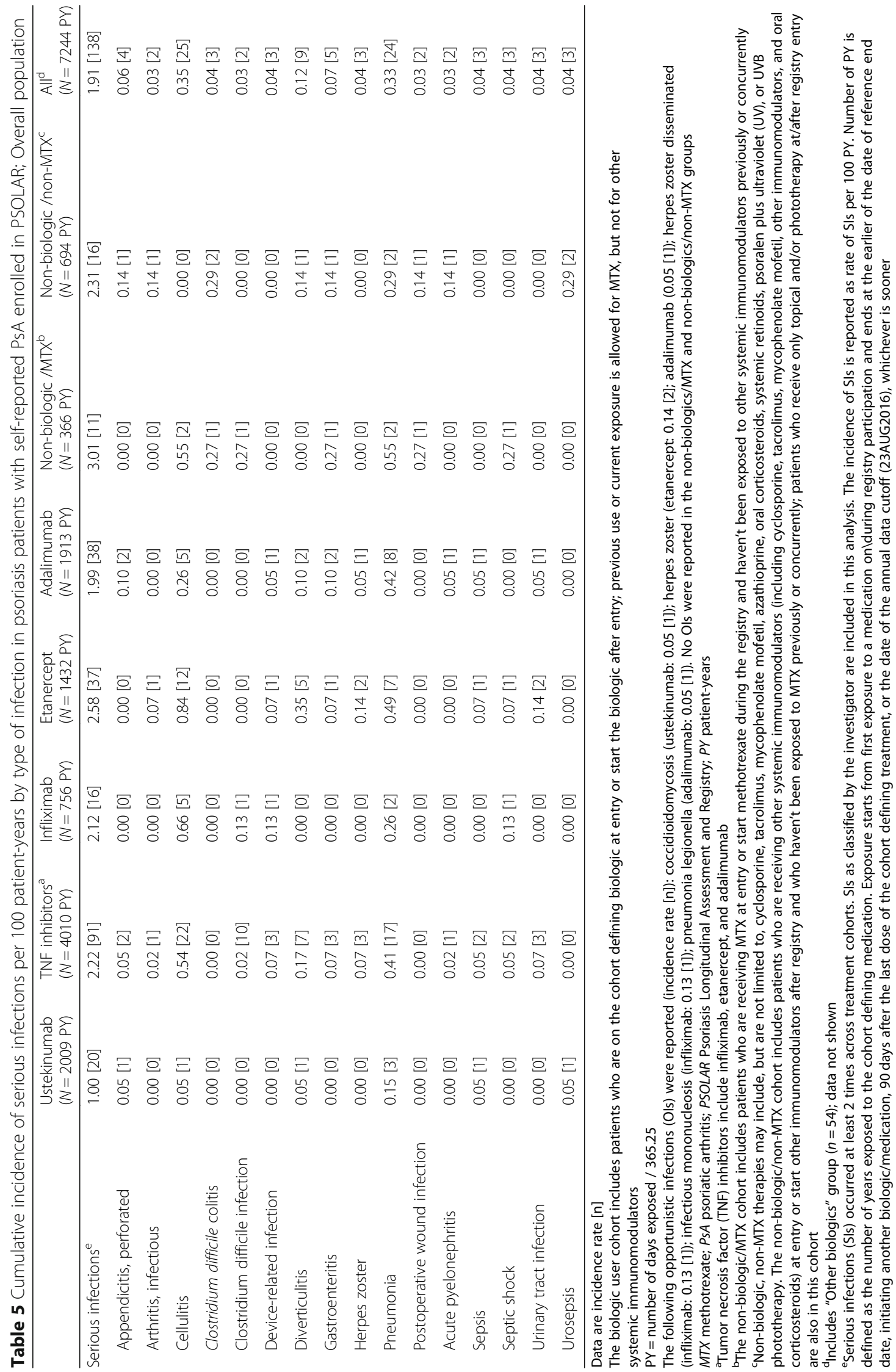


reported SIs among all patients, with the most cases of cellulitis $(n=22)$ and pneumonia $(n=17)$ both reported in the TNF inhibitors cohort (Table 5).

Multivariate analysis was used to determine predictors of time to first SI. Of the variables tested for the overall population, age, time-dependent disease activity PGA of 4, 5 (PGA scores of 4 or 5 at the time closest to the reported event), history of infection, and diabetes were associated $(p<0.05)$ with an increased risk for SI (Fig. 1). No increased risk for SI was observed with ustekinumab or non-biologic/nonMTX use. Findings were similar in the incident and bionaive populations; however, the number of patients in these cohorts was low and the 95\% CIs were wide (data not shown).

\section{Discussion}

Patients with PsA who are receiving biologics may have an elevated risk for developing SI. In this report, we used the PSOLAR psoriasis registry to evaluate 4315 psoriasis patients with self-reported PsA. This is the first modeled analysis of psoriasis patients with self-reported PsA enrolled in PSOLAR, the largest psoriasis registry to date.

It has been reported in several studies that TNF inhibitors are associated with an increased risk of SI in patients with psoriasis $[2,4-7]$ and in patients with PsA or RA $[8,21,22]$. Our results confirmed these findings among PSOLAR psoriasis patients self-reporting PsA [20]. These results are consistent with those previously reported for the overall PSOLAR population of patients with psoriasis, in which a higher risk of serious infections with the monoclonal TNF inhibitors compared with non-methotrexate and non-biologic therapies was found, with no increased risk observed with ustekinumab or etanercept [7]. With no adjustment, although not statistically significant, the SI rate was numerically higher in patients receiving TNF inhibitors and nonbiologics compared with patients receiving ustekinumab. After adjusting for potential confounders, the risk of SI for patients receiving ustekinumab or non-biologic/nonMTX was lower than MTX, while the risk of all TNF inhibitors was higher than MTX. However, none of the differences reached statistical significance $(p<0.05)$. The incidence rates of SIs in the non-biologic/non-MTX group were high (2.31); however, this cohort included patients who had received systemic corticosteroids. Rates of SIs in the overall population were similar to those reported in the incident population, but differed compared with the bionaive population in which the number of patients was low. Likewise, rates of SIs reported here were similar to those in PsA clinical trials with biologics $[8,17,23-25]$.
Currently, an unmet need remains for the treatment of patients with PsA. Most of the clinical and registry data available regarding safety pertains to patients with psoriasis or RA. Therefore, PSOLAR allows for the evaluation of a large population of patients with selfreported PsA, with greater than 12,000 patients registered, for a median of 5.11 years, and a total of 7244 PY of follow-up.

Patients with PsA often have other comorbidities thought to be associated with risk of infections. Several of the variables evaluated in this report, including age, time-dependent PGA 4, 5, history of infection, and diabetes were associated with a significantly increased risk for SI $(p<0.05)$. Interestingly, these variables were identical to those previously reported as being associated with SIs in the overall PSOLAR psoriasis population [7], thereby supporting our findings of psoriasis patients with self-reported PsA.

There are several limitations to consider here, as with any observational study. Treatment selection, recall, and reporting biases may have occurred, as these data are from a registry and not a clinical trial in which patients are randomized to treatment. PSOLAR longitudinally follows psoriasis patients; therefore, the subset of psoriasis patients with self-reported PsA could reflect potential treatment bias and may not reflect patients in the general rheumatology population. This is not a Classification Criteria for Psoriatic Arthritis defined PsA population, although up to approximately $30 \%$ of psoriasis patients may also develop concomitant PsA $[1,3]$. The fact that these patients also had psoriasis may have affected their medical history and influenced their therapy choices, thereby contributing to treatment bias. The increased use of biologics in this PsA subpopulation may be due to these patients having more active/severe psoriasis. Prior use of biologics was accounted for in the model; however, additional risks from previous therapies may not have been addressed. Additionally, patients in the non-biologic/non-MTX group only answered "yes" or "no" to the question of if they had received oral corticosteroids, with no details of dosing. In addition, these patients self-reported having PsA by completing an eCRF, with no further confirmation of their diagnosis by the investigator. Finally, some statistical differences were noted in patient characteristics across biologic cohorts at entry into the registry. Possible confounding characteristics were included in the multivariate model; however, there may be other characteristics that were not collected and therefore not accounted for in the model.

According to recent guidelines for the treatment of PsA, serious infections were chosen as one of the critical outcomes for comparisons between therapies and are noted to be one of the greatest concerns for patients and 
physicians when choosing among the currently available therapies [26]. The findings presented here could potentially inform and assist health care professionals when selecting an appropriate treatment option for their patients with PsA.

\section{Conclusions}

This analysis evaluated the incidence of SIs among the 2401 psoriasis patients with self-reported PsA in the overall population of the PSOLAR registry. Our results showed that rates of SIs were numerically higher among the self-reported PsA patients receiving TNF inhibitors (infliximab, adalimumab, or etanercept), but not in patients receiving ustekinumab; however, these differences were not statistically significant. Factors including increasing age, time-dependent PGA 4, 5, history of infection, and diabetes were also associated with an increased risk for SI in psoriasis patients with PsA. Continued follow-up of these PsA patients in the ongoing PSOLAR registry will provide additional safety information regarding patients with PsA in general.

\section{Supplementary information}

Supplementary information accompanies this paper at https://doi.org/10. 1186/s41927-019-0094-3.

Additional file 1: Table S1. Demographic and disease characteristics of psoriasis patients with self-reported PSA in the PSOLAR registry: Incident population.

Additional file 2: Table S2. Demographic and disease characteristics of psoriasis patients with self-reported PSA in the PSOLAR registry: Bionaive population.

\section{Abbreviations \\ AEs: Adverse events; Cl: Confidence interval; eCRF: Electronic case report forms; MedDRA: Medical Dictionary for Regulatory Activities; \\ MTX: Methotrexate; PSA: Psoriatic arthritis; PSOLAR: Psoriasis Longitudinal Assessment and Registry; PY: Patient years; RA: Rheumatoid arthritis; SAEs: Serious adverse events; SIs: Serious infections; TNF: Tumor necrosis factor}

\section{Acknowledgements \\ The authors wish to thank Kristin Ruley Sharples, PhD, of Janssen Scientific Affairs, LLC (Spring House, PA), for her writing and editorial support in the preparation of this manuscript, and Rongshuang Lin, PhD of PRA Health Sciences (Blue Bell, PA) for her statistical support.}

\section{Authors' contributions}

CTR, MS, YP, JB, SDC, SK, BS, WL, and ABG made substantial contributions to the study conception and design, acquisition of data, and analysis and interpretation of data; were involved in drafting the manuscript and revising it critically for important intellectual content; and gave final approval of the version to be published. CTR, MS, YP, JB, and ABG made substantial contributions to the collection and analysis of the data, were involved in revising manuscript content, and gave final approval of the version to be published. All authors participated sufficiently in the work to take public responsibility for appropriate portions of the content and agreed to be accountable for all aspects of the work by ensuring that questions related to the accuracy or integrity of any part of the work are appropriately investigated and resolved. All authors read and approved the final version of the manuscript for submission.

\section{Funding}

This study was funded by Janssen Biotech Inc., Horsham, PA, USA. The sponsor had a role in the design of the study and collection, analysis, and interpretation of data, and in writing the manuscript.

\section{Availability of data and materials}

The datasets generated and/or analyzed during the current study are not currently publicly available because the registry is still ongoing, but will be available by request or at clinicaltrials.gov (NCT00508547) when the registry concludes.

\section{Ethics approval and consent to participate}

PSOLAR is conducted in accordance with the International Conference on Harmonizing guidelines on Good Clinical Practices and the Declaration of Helsinki. An institutional review board or ethics committee (Goodwyn Institutional Review Board, and Ontario Institutional Review Board) approved the registry protocol. All patients provided written informed consent before initiation of study procedures and to publish the data.

\section{Consent for publication}

Not applicable.

\section{Competing interests}

C. T. Ritchlin has received consultant fees $(<\$ 10,000)$ from Amgen, Eli Lilly, Janssen, Pfizer, and UCB, and consultant fees $(>\$ 10,000)$ from AbbVie. M. Stahle has received consultant/speaking/honoraria $(<\$ 10,000)$ from AbbVie, Eli Lilly, Leo Pharma, and Novartis. Y. Poulin has received consultant/ speaking/ honoraria $(<\$ 10,000)$ from Amgen, Celgene, and Galderma, and fees $(>\$ 10,000)$ from AbbVie, Eli Lilly, Janssen, and Novartis. J. Bagel has received consultant fees $(<\$ 10,000)$ from Boehringer Ingelheim, and consultant/speaker/investigator fees $(>\$ 10,000)$ from Eli Lilly, Celgene, Janssen, Leo Pharma, Novartis, and Ortho Dermatologics. A. B. Gottlieb has received consultant/speaking/honoraria $(<\$ 10,000)$ from Beiersdorf, Merck, Novartis, and Valeant, and $(>\$ 10,000)$ from AbbVie, Eli Lilly, Janssen, Pfizer, and UCB. S. D. Chakravarty, S. Kafka, B. Srivastava, and W. Langholff are all employees of Janssen and own stock in Johnson \& Johnson, of which Janssen is a wholly-owned subsidiary.

\section{Author details}

'Division of Allergy, Immunology, and Rheumatology, University of Rochester Medical Center, 601 Elmwood Ave., Box 695, Rochester, NY 14642, USA. ${ }^{2}$ Department of Medicine, Karolinska University Hospital, Karolinska Institutet, Stockholm, Sweden. ${ }^{3}$ Université Laval and Centre de Recherche Dermatologique du Quebec métropolitain, Quebec, Canada. ${ }^{4}$ Psoriasis Treatment Center of Central New Jersey, East Windsor, NJ, USA. ${ }^{5}$ Janssen Scientific Affairs, LLC, Horsham, PA, USA. 'Drexel University College of Medicine, Philadelphia, PA, USA. ${ }^{7}$ Janssen Research \& Development, Spring House, PA, USA. ${ }^{8}$ New York Medical College, Metropolitan Hospital, New York, NY, USA

Received: 14 May 2019 Accepted: 10 October 2019 Published online: 28 November 2019

\section{References}

1. Gladman DD, Antoni C, Mease P, et al. Psoriatic arthritis: epidemiology, clinical features, course, and outcome. Ann Rheum Dis. 2005;64(Suppl 2): ii14-7.

2. Germano V, Cattaruzza MS, Osborn J, et al. Infection risk in rheumatoid arthritis and spondyloarthropathy patients under treatment with DMARDs, corticosteroids and TNF- $a$ antagonists. J Transl Med. 2014;12:77.

3. Ritchlin CT, Colbert RA, Gladman DD. Psoriatic arthritis. N Engl J Med. 2017; 376:957-70

4. Garcia-Doval I, Cohen AD, Cazzaniga S, et al. Psonet Network. Risk of serious infections, cutaneous bacterial infections, and granulomatous infections in patients with psoriasis treated with anti-tumor necrosis factor agents versus classic therapies: Prospective meta-analysis of Psonet registries. J Am Acad Dermatol. 2017;76:299-308.e16.

5. Dávila-Seijo P, Dauden E, Descalzo MA, et al. BIOBADADERM Study Group. Infections in moderate to severe psoriasis patients treated with biological drugs compared to classic systemic drugs: findings from the BIOBADADERM registry. J Invest Dermatol. 2017;137:313-21. 
6. Yiu ZZ, Exton LS, Jabbar-Lopez Z, et al. Risk of serious infections in patients with psoriasis on biologic therapies: a systematic review and meta-analysis. J Invest Dermatol. 2016;136:1584-91.

7. Kalb RE, Fiorentino DF, Lebwohl MG, et al. Risk of serious infection with biologic and systemic treatment of psoriasis: results from the Psoriasis Longitudinal Assessment and Registry (PSOLAR). JAMA Dermatol. 2015;151: 961-9.

8. Minozzi S, Bonovas S, Lytras T, et al. Risk of infections using anti-TNF agents in rheumatoid arthritis, psoriatic arthritis, and ankylosing spondylitis: a systematic review and meta-analysis. Expert Opin Drug Saf. 2016;15(sup1): 11-34.

9. Accortt NA, Bonafede MM, Collier DH, et al. Risk of subsequent infection among patients receiving tumor necrosis factor inhibitors and other disease-modifying antirheumatic drugs. Arthritis Rheumatol. 2016;68:67-76

10. Singh JA, Cameron C, Noorbaloochi S, et al. Risk of serious infection in biological treatment of patients with rheumatoid arthritis: a systematic review and meta-analysis. Lancet. 2015;386:258-65.

11. Subesinghe S, Rutherford Al, Byng-Maddick R, et al. Recurrent serious infections in patients with rheumatoid arthritis-results from the British Society for Rheumatology Biologics Register. Rheumatology (Oxford). 2018, 57:651-5.

12. Richter A, Listing J, Schneider M, et al. Impact of treatment with biologic DMARDs on the risk of sepsis or mortality after serious infection in patients with rheumatoid arthritis. Ann Rheum Dis. 2016;75:1667-73.

13. Haddad A, Li S, Thavaneswaran A, et al. The incidence and predictors of infection in psoriasis and psoriatic arthritis: results from longitudinal observational cohorts. J Rheumatol. 2016;43:362-6.

14. Pérez-Plaza A, Carretero G, Ferrandiz C, et al. Biobadaderm Study Group. Comparison of phenotype, comorbidities, therapy and adverse events between psoriatic patients with and without psoriatic arthritis. Biobadaderm registry. J Eur Acad Dermatol Venereol. 2017;31:1021-8.

15. Ramiro S, Gaujoux-Viala C, Nam JL, et al. Safety of synthetic and biological DMARDs: a systematic literature review informing the 2013 update of the EULAR recommendations for management of rheumatoid arthritis. Ann Rheum Dis. 2014;73:529-35.

16. Galloway JB, Hyrich KL, Mercer LK, et al. BSRBR Control Centre Consortium; British Society for Rheumatology Biologics Register. Anti-TNF therapy is associated with an increased risk of serious infections in patients with rheumatoid arthritis especially in the first 6 months of treatment: updated results from the British Society for Rheumatology Biologics Register with special emphasis on risks in the elderly. Rheumatology (Oxford). 2011;50: 124-31.

17. Dommasch ED, Abuabara K, Shin DB, et al. The risk of infection and malignancy with tumor necrosis factor antagonists in adult patients with psoriatic disease: a systematic review and meta-analysis of randomized controlled trials. J Am Acad Dermatol. 2011;64:1035-50.

18. Papp KA, Strober B, Augustin M, et al. PSOLAR investigators and steering committee. PSOLAR: design, utility, and preliminary results of a prospective, international, disease-based registry of patients with psoriasis who are receiving, or are candidates for, conventional systemic treatments or biologic agents. J Drugs Dermatol. 2012;11:1210-7.

19. Gottlieb AB, Kalb RE, Langley RG, et al. Safety observations in 12095 patients with psoriasis enrolled in an international registry (PSOLAR): experience with infliximab and other systemic and biologic therapies. J Drugs Dermatol. 2014;13:1441-8.

20. Kavanaugh A, Papp K, Gottlieb AB, et al. Demography, baseline disease characteristics, and treatment history of psoriasis patients with self-reported psoriatic arthritis enrolled in the PSOLAR registry. BMC Rheumatology. 2018; 2:29.

21. Dixon WG, Watson K, Lunt M, et al. British Society for Rheumatology biologics register. Rates of serious infection, including site-specific and bacterial intracellular infection, in rheumatoid arthritis patients receiving anti-tumor necrosis factor therapy: results from the British Society for Rheumatology Biologics Register. Arthritis Rheum. 2006;54:2368-76.

22. Gómez-Reino JJ, Carmona L, Valverde VR, et al. BIOBADASER group. Treatment of rheumatoid arthritis with tumor necrosis factor inhibitors may predispose to significant increase in tuberculosis risk: a multicenter activesurveillance report. Arthritis Rheum. 2003;48:2122-7.

23. Ritchlin C, Rahman P, Kavanaugh A, et al. PSUMMIT 2 Study Group. Efficacy and safety of the anti-IL-12/23 p40 monoclonal antibody, ustekinumab, in patients with active psoriatic arthritis despite conventional non-biological and biological anti-tumour necrosis factor therapy: 6-month and 1-year results of the phase 3, multicentre, double-blind, placebo-controlled, randomised PSUMMIT 2 trial. Ann Rheum Dis. 2014;73:990-9.

24. Mease PJ, van der Heijde D, Ritchlin CT, et al. SPIRIT-P1 Study Group. Ixekizumab, an interleukin-17A specific monoclonal antibody, for the treatment of biologic-naïve patients with active psoriatic arthritis: results from the 24-week randomised, double-blind, placebo-controlled and active (adalimumab)-controlled period of the phase III trial SPIRIT-P1. Ann Rheum Dis. 2017:76:79-87.

25. Nash P, Kirkham B, Okada M, et al. SPIRIT-P2 study group. Ixekizumab for the treatment of patients with active psoriatic arthritis and an inadequate response to tumour necrosis factor inhibitors: results from the 24-week randomised, double-blind, placebo-controlled period of the SPIRIT-P2 phase 3 trial. Lancet. 2017;389:2317-27.

26. Singh JA, Guvatt G, Ogdie A, et al. Special article: 2018 American College of Rheumatology/National Psoriasis Foundation guideline for the treatment of psoriatic arthritis. Arthritis Rheumatol. 2019;71:5-32.

\section{Publisher's Note}

Springer Nature remains neutral with regard to jurisdictional claims in published maps and institutional affiliations.
Ready to submit your research? Choose BMC and benefit from:

- fast, convenient online submission

- thorough peer review by experienced researchers in your field

- rapid publication on acceptance

- support for research data, including large and complex data types

- gold Open Access which fosters wider collaboration and increased citations

- maximum visibility for your research: over $100 \mathrm{M}$ website views per year

At BMC, research is always in progress.

Learn more biomedcentral.com/submissions 